\title{
KOEFFIZIENTENABSCHÄTZUNGEN FÜR FUNKTIONEN, DEREN ERSTE ABLEITUNG BESCHRÄNKTES ARGUMENT HAT
}

\author{
KARL DOPPEL und LUTZ VOLKMANN
}

Herrn Professor Dr. Albert Pfluger in Verehrung gewidmet

1. Einleitung und Ergebnisse. Viele im offenen Einheitskreis $|z|<1$ analytische Funktionen $f$ besitzen eine Darstellung

$$
\log f^{\prime}(z)=-\frac{1}{\pi} \int_{0}^{2 \pi} \log \left(1-e^{-i t} z\right) d \mu(t) .
$$

Ist $\mu$ etwa eine Treppenfunktion mit endlich vielen Sprüngen, so stellt (1) die bekannte Schwarz-Christoffelsche Formel dar. Im Fall, daß $\mu$ eine monoton wachsende Funktion mit $\mu(2 \pi)-\mu(0)=2 \pi$ ist, erklärt (1) eine konvexe Funktion. Gilt schließlich für $\mu$

$$
\int_{0}^{2 \pi} d \mu(t)=2 \pi \quad \text { und } \quad \int_{0}^{2 \pi}|d \mu(t)| \leqq k \pi
$$

mit einer reellen Zahl $k \geqq 2$, so wird durch (1) gerade eine Funktion $f$ beschränkter Randdrehung aus der Klasse $V_{k}$ definiert (man vergleiche dazu [6] und [7]). Integriert man (1) partiell, so erhält man

$$
\log f^{\prime}(z)=\frac{1}{\pi} \int_{0}^{2 \pi} \mu(t) d \log \left(1-e^{-i t} z\right)
$$

Wie aus dieser Form ersichtlich ist, ergibt sich die Möglichkeit, allgemeinere Funktionen $\mu$ als in der Darstellung (1) zuzulassen. Es sei $x>0$ vorgegeben. $\mathrm{Zu}$ jeder reellen Funktion $\mu$, die den Bedingungen

$$
\begin{gathered}
\mu \in L^{\infty}[0,2 \pi], \\
\int_{0}^{2 \pi} \mu(t) d t=0 \quad \text { und } \quad \underset{0 \leqq t \leqq 2 \pi}{\operatorname{ess} \sup }|\mu(t)| \leqq \pi \varkappa
\end{gathered}
$$

genügt, wird durch (2) eine im offenen Einheitskreis $|z|<1$ analytische Funktion $f$ der Gestalt

$$
f(z)=z+\sum_{n=2}^{\infty} a_{n} z^{n}
$$


mit der Eigenschaft

$$
\left|\arg f^{\prime}(z)\right| \leqq \pi \varkappa \quad(|z|<1)
$$

erklärt. Umgekehrt läßt sich jede Funktion (4) mit der Eigenschaft (4a) als ein Lebesgue-Stieltjessches Integral (2) mit einer Funktion $\mu$, die die Bedingungen (3a) und (3b) erfüllt, schreiben (man vergleiche [3]). Die Klasse dieser Funktionen $f$ wird im folgenden mit $H_{\varkappa}$ bezeichnet.

Bekanntlich sind für die konvexen Funktionen (man vergleiche [4], S. 172, oder [9], S. 46) sowie für die Funktionen beschränkter Randdrehung (man vergleiche [1], [2], [5] und [10], S. 26) Koeffizientenabschätzungen gegeben worden. In dieser Note werden nun Abschätzungen für die Koeffizienten der Funktionen $f$ der Klasse $H_{\varkappa}$ hergeleitet, wobei die Existenz von maximalen Koeffizienten $\left|a_{n}(f)\right|$ in dieser Klasse durch folgende Überlegung gesichert ist:

In [3] wurde für alle $f$ aus $H_{x}$ gezeigt:

Daraus folgt nun wegen

$$
\left(\frac{1-|z|}{1+|z|}\right)^{2 \varkappa} \leqq\left|f^{\prime}(z)\right| \leqq\left(\frac{1+|z|}{1-|z|}\right)^{2 \varkappa}
$$

$$
|f(z)| \leqq \int_{0}^{z}\left|f^{\prime}(z)\right||d z|,
$$

daß $H_{x}$ eine lokal gleichmäßig beschränkte und daher nach dem Satz von Montel eine normale Familie bildet. Ist nun $\left(f_{n}\right)$ eine Folge von Funktionen aus $H_{\varkappa}$ mit $f(z)=\lim _{n \rightarrow \infty} f_{n}(z)$, so folgt $f^{\prime}(z)=\lim _{n \rightarrow \infty} f_{n}^{\prime}(z)$ und daher $f(0)=0$ und $f^{\prime}(0)=1$. Aus (5) ergibt sich $f^{\prime}(z) \neq 0$ und weiter $\left|\arg f^{\prime}(z)\right| \leqq \pi x$, womit $H_{x}$ eine kompakte Familie ist (man vergleiche [8], § 14).

In der vorliegenden Note werden für die Koeffizienten der Funktionen $f$ aus $H_{x}$ die scharfen Abschätzungen

$$
\begin{gathered}
\left|a_{n}\right| \leqq \frac{4 \varkappa}{n} \quad \text { für } \quad x \leqq \frac{1}{2} \\
\left|a_{n}\right| \leqq \frac{1}{n} \sum_{k=0}^{n-1}\left(\begin{array}{c}
2 \varkappa \\
n-1-k
\end{array}\right)\left(\begin{array}{c}
2 x+k-1 \\
k
\end{array}\right) \text { für } x>\frac{1}{2}
\end{gathered}
$$

bewiesen.

Die Verfasser möchten Herrn I. S. Louhivaara für wertvolle Hinweise und Bemerkungen danken.

2. Der Fall $x \leqq 1 / 2$. Für die folgenden Untersuchungen wird die vorher angegebene geometrische Charakterisierung der Funktionen aus $H_{x}$ zugrunde gelegt, d. h. wir betrachten für $x>0$ die Menge der im offenen Einheitskreis $|z|<1$ lokal schlichten Funktionen $f$ der Gestalt

$$
f(z)=z+\sum_{n=2}^{\infty} a_{n} z^{n} \quad(|z|<1)
$$

mit der Eigenschaft

$$
\left|\arg f^{\prime}(z)\right| \leqq \pi \varkappa \quad(|z|<1) .
$$


Satz 1. Ist $f$ aus $H_{\varkappa}$ mit $\varkappa \leqq 1 / 2$, so gilt für alle $n \geqq 2$

$$
\left|a_{n}\right| \leqq \frac{4 \varkappa}{n} .
$$

Beweis. Für eine feste natürliche Zahl $n$ setze man

$$
\begin{aligned}
h(z) & =\frac{1}{n} \sum_{v=1}^{n} f^{\prime}\left(z e^{2 \pi i v / n}\right) \\
& =1+\sum_{j=1}^{\infty}(j+1) a_{j+1} z^{j} \frac{1}{n} \sum_{v=1}^{n} e^{2 \pi i j v / n} \\
& =1+\sum_{v=1}^{\infty}(n v+1) a_{n v+1} z^{n v} \\
& =1+(n+1) a_{n+1} z^{n}+\ldots
\end{aligned}
$$

Wegen (4a) liegen die $n$ Punkte $\zeta_{v}=f^{\prime}\left(z e^{2 \pi i v / n}\right) \quad(v=1,2, \ldots, n)$ im Winkelraum $|\arg z| \leqq \pi \varkappa$. Da nach Voraussetzung $\chi \leqq 1 / 2$ ist, liegt auch das arithmetische Mittel der Punkte $\zeta_{v}$ im Winkelraum $|\arg z| \leqq \pi \varkappa$, und daher gilt wegen (7)

$$
|\arg h(z)| \leqq \pi \varkappa
$$

für alle $|z|<1$.

Man setze nun

Dann folgt wegen (7)

$$
g(z)=z \frac{h^{\prime}(z)}{h(z)}
$$

und weiter $\left(z=r e^{i \theta}\right)$

$$
g(z)=n(n+1) a_{n+1} z^{n}+\sum_{k=n+1}^{\infty} b_{k} z^{k}
$$

mit

$$
g(z)=\sum_{k=n}^{\infty} b_{k} r^{k}(\cos k \theta+i \sin k \theta)
$$

$$
b_{n}=n(n+1) a_{n+1} .
$$

Multipliziert man (9) mit $\cos n \theta$ bzw. $\sin n \theta$ und integriert danach gliedweise, so erhält man

bzw.

$$
b_{n} r^{n}=\frac{1}{\pi} \int_{0}^{2 \pi} g\left(r e^{i \theta}\right) \cos n \theta d \theta
$$

$$
i b_{n} r^{n}=\frac{1}{\pi} \int_{0}^{2 \pi} g\left(r e^{i \theta}\right) \sin n \theta d \theta .
$$

Wegen $b_{n}=\operatorname{Re} b_{n}-i \operatorname{Re}\left(i b_{n}\right)$ folgt daraus

$$
b_{n}=\frac{1}{\pi r^{n}} \int_{0}^{2 \pi} \operatorname{Re}\left\{g\left(r e^{i \theta}\right)\right\} e^{-i n \theta} d \theta .
$$


Beachtet man

so ergeben (9) und (10)

$$
\operatorname{Re}\left\{r e^{i \theta} \frac{h^{\prime}\left(r e^{i \theta}\right)}{h\left(r e^{i \theta}\right)}\right\}=\frac{\partial}{\partial \theta} \arg h\left(r e^{i \theta}\right),
$$

$$
\begin{aligned}
b_{n} & =\frac{1}{\pi r^{n}} \int_{0}^{2 \pi} \frac{\partial}{\partial \theta} \arg h\left(r e^{i \theta}\right) e^{-i n \theta} d \theta \\
& =\frac{i n}{\pi r^{n}} \int_{0}^{2 \pi} \arg h\left(r e^{i \theta}\right) e^{-i n \theta} d \theta .
\end{aligned}
$$

Setzt man $-\arg b_{n}=\beta$, so folgt daraus

$$
\begin{aligned}
\left|b_{n}\right| & =b_{n} e^{i \beta}=\frac{i n}{\pi r^{n}} \int_{0}^{2 \pi} \arg h\left(r e^{i \theta}\right) e^{i(\beta-n \theta)} d \theta \\
& =\frac{-n}{\pi r^{n}} \int_{0}^{2 \pi} \arg h\left(r e^{i \theta}\right) \sin (\beta-n \theta) d \theta
\end{aligned}
$$

und unter Berücksichtigung von (8)

$$
\left|b_{n}\right| \leqq \frac{n}{r^{n}} \varkappa \int_{0}^{2 \pi}|\sin (\beta-n \theta)| d \theta \leqq \frac{4 n \varkappa}{r^{n}} .
$$

Beachtet man (9a), so folgt schließlich mit $r \rightarrow 1$

also

$$
\left|n(n+1) a_{n+1}\right|=\left|b_{n}\right| \leqq 4 n \%,
$$

$$
\left|a_{n+1}\right| \leqq \frac{4 \%}{n+1}
$$

womit (6a) bewiesen ist.

3. Der Fall $x \geqq 1 / 2$. Im Fall $x \geqq 1 / 2$ gelten folgende Koeffizientenabschätzungen.

Satz 2. Ist f aus $H_{\varkappa}$ mit $x \geqq 1 / 2$, so gilt für alle $n \geqq 2$

$$
\left|a_{n}\right| \leqq \frac{1}{n} \sum_{k=0}^{n-1}\left(\begin{array}{c}
2 \varkappa \\
n-1-k
\end{array}\right)\left(\begin{array}{c}
2 \varkappa+k-1 \\
k
\end{array}\right) .
$$

Beweis. Für beliebiges $f$ aus $H_{\varkappa}$ gilt

und somit

$$
\left|\arg f^{\prime}(z)\right| \leqq \pi \% \quad(|z|<1)
$$

$$
\left|\arg \left(f^{\prime}(z)\right)^{1 /(2 x)}\right|=\frac{1}{2 \varkappa} \arg f^{\prime}(z) \mid \equiv \frac{\pi}{2} \quad(|z|<1)
$$

Daher ist die Funktion $p$,

$$
p(z)=\left(f^{\prime}(z)\right)^{1 /(2 x)} \quad(|z|<1)
$$

eine im offenen Einheitskreis analytische Funktion mit positivem Realteil. Da $p$ durch die Funktion $q$,

$$
q(z)=\frac{1+z}{1-z} \quad(|z|<1)
$$


subordiniert wird (man vergleiche [9], S. 36) folgt nach einem Theorem von Brannan, Clunie und Kirwan (man vergleiche [2], S. 8, oder [10], S. 17), daß für jedes reelles $\alpha \geqq 1$ die Koeffizienten von $p^{\alpha}$ durch die entsprechenden Koeffizienten von $q^{\alpha}$ dominiert werden. Beachtet man

$$
\left(\frac{1+z}{1-z}\right)^{2 \varkappa}=1+\sum_{n=1}^{\infty}\left\{\sum_{k=0}^{n}\left(\begin{array}{c}
2 \varkappa \\
n-k
\end{array}\right)\left(\begin{array}{c}
2 \varkappa+k-1 \\
k
\end{array}\right)\right\} z^{n} \quad(|z|<1)
$$

und

so folgt wegen $2 x \geqq 1$

$$
(p(z))^{2 \varkappa}=f^{\prime}(z)=1+\sum_{n=2}^{\infty} n a_{n} z^{n-1} \quad(|z|<1),
$$

$$
n\left|a_{n}\right| \leqq \sum_{k=0}^{n-1}\left(\begin{array}{c}
2 \varkappa \\
n-1-k
\end{array}\right)\left(\begin{array}{c}
2 \varkappa+k-1 \\
k
\end{array}\right),
$$

womit Satz 2 bewiesen ist.

4. Die Schärfe der Abschätzungen. Im Fall $x \geqq 1 / 2$ leistet die Funktion $f_{\varkappa, t}$, $t$ beliebig reell

$$
f_{\varkappa, \iota}(z)=z+\sum_{n=2}^{\infty}\left\{\frac{e^{-i(n-1) t}}{n} \sum_{k=0}^{n-1}\left(\begin{array}{c}
2 \varkappa \\
n-1-k
\end{array}\right)\left(\begin{array}{c}
2 \varkappa+k-1 \\
k
\end{array}\right)\right\} z^{n}
$$

das Gewünschte (man vergleiche [3]).

Im Fall $0<\varkappa \leqq 1 / 2$ betrachte man für $n \geqq 2$ die Funktionen $f_{n, \varkappa}$,

$$
f_{n, x}(z)=\int_{0}^{z}\left(\frac{1+\zeta^{n-1}}{1-\zeta^{n-1}}\right)^{2 \varkappa} d \zeta \quad(|z|<1) .
$$

Wegen

gilt offensichtlich

$$
\left|\arg \left\{\frac{1+z^{n-1}}{1-z^{n-1}}\right\}\right| \leqq \frac{\pi}{2}
$$

$$
\left|\arg f_{n, \varkappa}^{\prime}(z)\right| \leqq \pi \varkappa
$$

und folglich gehört $f_{n, \varkappa}$ zu $H_{\varkappa}$. Andererseits findet man sofort

$$
f_{n, x}(z)=z+\frac{4 \varkappa}{n} z^{n}+\ldots \quad(|z|<1),
$$

so daß für jedes $n \geqq 2$ jeweils der $n$-te Koeffizient der Funktion $f_{n, \varkappa}$ bezüglich Satz 1 extremal ist. ${ }^{1}$ )

1) Im Gegensatz zu den Funktionen mit beschränkter Randdrehung gilt für die in dieser Note betrachtete Funktionenklasse folgender Sachverhalt:

Ist $f$ aus $H_{\varkappa}$ und $x \leqq 1$, so gelten für die $n$-ten Koeffizienten $a_{n}(f)$ die Abschätzungen

aber die Funktionen $f_{3, \varkappa}$,

$$
\left|a_{n}(f)\right| \leqq n, \quad n=2,3, \ldots,
$$

$$
f_{3, \varkappa}(z)=\int_{0}^{z}\left(\frac{1+\zeta^{2}}{1-\zeta^{2}}\right)^{2 \varkappa} d \zeta \quad(|z|<1)
$$

sind für alle $x>3 / 4$ nicht schlicht (man vergleiche [9], Theorem 1.5). 
5. Ein Verzerrungssatz. In [3] wurde für alle $f$ aus $H_{x}$ und alle $|z|<1$ bewiesen:

und

$$
\left(\frac{1-|z|}{1+|z|}\right)^{2 \varkappa} \leqq\left|f^{\prime}(z)\right| \leqq\left(\frac{1+|z|}{1-|z|}\right)^{2 \varkappa}
$$

$$
\left|f^{\prime \prime}(z)\right| \leqq 2 \pi \varkappa\left(\frac{1+|z|}{1-|z|}\right)^{2 \varkappa} \frac{1}{1-|z|^{2}} .
$$

Da für die in (12) angegebene Funktion $f_{\varkappa, t}$ gilt:

$$
f_{\varkappa, t}^{\prime}(z)=\left(\frac{1+e^{-i t} z}{1-e^{-i t} z}\right)^{2 \varkappa}
$$

liefert (5) die scharfen Schranken für die Verzerrung von $f$ (man vergleiche [3]). Im folgenden Satz werden für die $m$-ten Ableitungen $(m \geqq 2)$ von $f$ scharfe (obere) Schranken angegeben, falls $x \geqq 1 / 2$ ist.

Satz 3. Ist $f$ aus $H_{x}$ mit $x \geqq 1 / 2$, so gilt für alle $m \geqq 2$ und alle $|z|<1$

$$
\left|f^{(m)}(z)\right| \leqq \frac{d^{m-1}}{d|z|^{m-1}}\left(\frac{1+|z|}{1-|z|}\right)^{2 x} .
$$

Beweis. Setzt man

$$
\left(\frac{1+z}{1-z}\right)^{2 \varkappa}=\sum_{n=0}^{\infty} A_{n} z^{n}
$$

so sind alle $A_{n}$ reell und positiv (man vergleiche (11)). Setzt man

$$
f^{\prime}(z)=\sum_{n=0}^{\infty} \alpha_{n} z^{n}
$$

so gilt wegen Satz 2 für alle $n$

$$
\left|\alpha_{n}\right| \leqq A_{n}
$$

Für $m \geqq 2$ folgt aus (15)

$$
\frac{d^{m-1}}{d|z|^{m-1}}\left(\frac{1+|z|}{1-|z|}\right)^{2 \varkappa}=\sum_{n=m-1}^{\infty} n(n-1) \ldots(n-m+2) A_{n}|z|^{n-m+1}
$$

und aus (16) und (17)

$$
\begin{aligned}
\left|f^{(m)}(z)\right| & =\left|\sum_{n=m-1}^{\infty} n(n-1) \ldots(n-m+2) \alpha_{n} z^{n-m+1}\right| \\
& \leqq \sum_{n=m-1}^{\infty} n(n-1) \ldots(n-m+2)\left|\alpha_{n}\right||z|^{n-m+1} \\
& \leqq \sum_{n=m-1}^{\infty} n(n-1) \ldots(n-m+2) A_{n}|z|^{n-m+1}
\end{aligned}
$$

Aus (18) und (19) folgt sofort die gewünschte Abschätzung (14). 
Bemerkungen. Die in (13) angegebene Funktion $f_{x, 0}^{\prime}$ definiert durch

$$
f_{\varkappa, 0}^{\prime}(z)=\left(\frac{1+z}{1-z}\right)^{2 \varkappa}
$$

liefert für $z=|z|$ in (14) das Gleichheitszeichen, womit diese Ungleichung scharf ist. Für den Fall $x<1 / 2$ bleibt die Frage nach der scharfen Abschätzung der $m$-ten Ableitung von $f(m \geqq 2)$ offen.

\section{Literatur}

[1] Aharonov, D., und S. Friedland: On an inequality connected with the coefficient conjecture for functions of bounded boundary rotation. - Ann. Acad. Sci. Fenn. Ser. A. I. Math. $524,1973,1-13$.

[2] Brannan, D. A., J. G. Clunie, und W. E. Kirwan: On the coefficient problem for functions of bounded boundary rotation. - Ann. Acad. Sci. Fenn. Ser. A. I. Math. 523, 1973, $1-18$.

[3] Doppel, K.: Über lokal schlichte Funktionen, deren erste Ableitung beschränktes Argument hat. - Ann. Acad. Sci. Fenn. Ser. A. I. Math. 3, 1977, 317-325.

[4] Golusin, G. M.: Geometrische Funktionentheorie. - Hochschulbücher für Mathematik 31. VEB Deutscher Verlag der Wissenschaften, Berlin, 1957.

[5] Lehto, O.: On the distortion of conformal mappings with bounded boundary rotation. - Ann. Acad. Sci. Fenn. Ser. A. I. Math.-Phys. 124, 1952, 1-14.

[6] LöWNER, K.: Untersuchungen über die Verzerrung bei konformen Abbildungen des Einheitskreises $|z|<1$, die durch Funktionen mit nicht verschwindender Ableitung geliefert werden. - Berichte über die Verhandlungen der Königl. Sächsischen Gesellschaft der Wissenschaften zu Leipzig, Mathematisch-physikalische Klasse 69, 1917, 89_ 106.

[7] PaAtero, V.: Über die konforme Abbildung von Gebieten, deren Ränder von beschränkter Drehung sind. - Ann. Acad. Sci. Fenn. Ser. A. 33:9, 1931, 1-78.

[8] Pfluger, A.: Lectures on conformal mapping. - [Vorlesungsausarbeitung.] Indiana University, Department of Mathematics, Bloomington, 1969.

[9] Pommerenke, Chr.: Univalent functions. - Mathematische Lehrbücher 25. Vandenhoeck und Ruprecht, Göttingen, 1975.

[10] Schober, G.: Univalent functions - Selected topics. - Lecture Notes in Mathematics 478 , Springer-Verlag, Berlin-Heidelberg-New York, 1975.

Freie Universität Berlin

I. Mathematisches Institut

Hüttenweg 9

D 1000 Berlin 33

Eingegangen am 17. November 1978 